\title{
Practical Network-Based Techniques for Mobile Positioning in UMTS
}

\author{
Jakub Borkowski and Jukka Lempiäinen \\ Institute of Communications Engineering, Tampere University of Technology, P.O. Box 553, 33101 Tampere, Finland
}

Received 1 June 2005; Revised 9 May 2006; Accepted 18 May 2006

\begin{abstract}
This paper presents results of research on network-based positioning for UMTS (universal mobile telecommunication system). Two new applicable network-based cellular location methods are proposed and assessed by field measurements and simulations. The obtained results indicate that estimation of the position at a sufficient accuracy for most of the location-based services does not have to involve significant changes in the terminals and in the network infrastructure. In particular, regular UMTS terminals can be used in the presented PCM (pilot correlation method), while the other proposed method - the ECID+RTT (cell identification + round trip time) requires only minor software updates in the network and user equipment. The performed field measurements of the PCM reveal that in an urban network, $67 \%$ of users can be located with an accuracy of $70 \mathrm{~m}$. In turn, simulations of the ECID+RTT report accuracy of $60 \mathrm{~m}-100 \mathrm{~m}$ for $67 \%$ of the location estimates in an urban scenario.
\end{abstract}

Copyright (c) 2006 J. Borkowski and J. Lempiäinen. This is an open access article distributed under the Creative Commons Attribution License, which permits unrestricted use, distribution, and reproduction in any medium, provided the original work is properly cited.

\section{INTRODUCTION}

An ultimate aim of the mobile positioning research is to find a method providing high estimation accuracy to the user with minimum delay and at minimum cost. Development of location techniques towards defined performance objectives is pushed by the perspective of high revenues through enabling attractive location-sensitive applications together with stated safety requirements. Currently, the best positioning accuracy is provided by the AGPS (assisted global positioning system) method [1]. However, this technique has the highest hardware constraints, as UMTS (universal mobile telecommunications system) mobiles in the current market are not typically AGPS-enabled. Moreover, most of the existing UMTS networks are not ready for AGPS positioning technology, since upgrade of present equipment and implementation of additional units such as LMU (location measurement unit) is needed. Naturally, reducing the required investments for deploying technology that enables positioning with sufficient accuracy is essential in providing LBS (location-based services). Therefore, from this perspective, a motivation for cellular location techniques that are ready for immediate deployment is magnified. Positioning techniques that do not require major changes in network and in terminal and utilize only existing network infrastructure to provide a location of the user could be directly implemented in the current networks to provide a wide range of LBS. In the long-term deployment, the cellular positioning methods could be used as supporting techniques for AGPS when the availability of more accurate and complex systems will considerably increase. Hence, the latency, accuracy, and indoor availability of the satellite-based positioning will be significantly enhanced, resulting in more reliable position estimation for the end user.

The aim of this paper is to present two applicable network-based cellular positioning techniques for UMTS. They are ECID+RTT (enhanced cell identification + round trip time) [2,3] and PCM (pilot correlation method) [4]. The proposed positioning methods are based entirely on standardized messages and procedures. They do not require implementation of LMUs, since the network synchronization is not mandatory. Moreover, the overall requirement of network and terminal modification is kept at the minimum possible level, placing the applicability of the ECID+RTT and PCM at a high level. The performance of the developed location methods is evaluated by measurement campaigns performed in an urban and suburban UMTS network as well as by simulations. In addition, impact of positioning on network capacity is assessed by field measurements. 


\section{CALL FOR POSITIONING}

Development of positioning techniques for cellular networks was mainly motivated by emergency requirements stating that all 911 calls in the United States need to be located with a certain level of accuracy. The FCC (Federal Communication Commission) report for Phase II issued in 1999 imposes that cellular carriers need to have network-based capabilities to estimate the location of the user with the accuracy of $100 \mathrm{~m}$ for $67 \%$ of calls and $300 \mathrm{~m}$ for $95 \%$ of calls [5]. In turn, the minimum required accuracy for mobile-based positioning solutions is $50 \mathrm{~m}$ for $67 \%$ of calls and $150 \mathrm{~m}$ for $95 \%$ of calls. Such accuracy requirements should have been provided by location technologies available not later than by October 2001. Moreover, FCC also regulates the expected penetration of positioning capable terminals in the North American market. Network operators were obligated to ensure that with the beginning of 2005, $100 \%$ penetration of positioning-enabled terminals in their subscriber base should have been achieved.

In Europe, the European Commission has taken initiatives. This organization has established the Coordination Group on Access to Location Information by Emergency Services with the aim to define requirements for common location providing mechanism that can be accessible by the European 112 community and emergency service operators. However, in Europe as well as in the Far East markets, it has been observed that greater emphases are placed on commercial applications [6].

Location-sensitive applications can be generally classified to pull, push, and track services. Pull applications require the user to send a request for information that is sensitive to the current location of the subscriber. Examples of such value-added services constitute location of the nearest interest point (e.g., mobile yellow pages). The required accuracy of position estimation for beneficial operation of most of such services is at the level of $100 \mathrm{~m}$ for $67 \%$ of requests [7]. In turn, push-type services send adequate information to the subscriber depending on his location or location of defined objects without the need of sending separate enquiries. In the case of commercial push-type applications, the subscriber can be notified, for instance, about the position of the defined person or about the actual offers of businesses in the current area (localized advertising). Similarly, based on the user location, certain roadside assistance can be provided. Push-type applications also include various connecting interactive services such as location-sensitive games or area chat rooms. Emergency services can be categorized as push-type LBS as well, however, in this case, the user is not informed about its location but naturally the position of the caller is forwarded directly to responsible organization. Correspondingly, most of the commercial push applications do not require high positioning accuracy, that is, below $100 \mathrm{~m}$ for $67 \%$ of estimates [7]. The third category constitutes a tracking type of LBS. These services permanently report the position of the object (e.g., car navigation, fleet management, etc.). Most of referred services do not require high estimation accuracy. However, there are examples, for instance, route guidance for the blind, where the accuracy at submeter level is needed.
Availability of location information can significantly improve the functionality of RRM (radio resource management) in cellular networks. Location-sensitive handover schemes that avoid frequent handovers of users at the cell edge areas or provide intelligent assignment of users to the cell in HCS (hierarchical cell structure) are just the selected examples of possible exploitation of location information [810]. Moreover, provision of the caller position allows operator to apply more flexible charging schemes, for instance, home-zone billing approach.

\section{AN OVERVIEW OF EXISTING LOCATION TECHNOLOGIES}

Three major location techniques for UMTS have been specified in the 3GPP (Third-Generation Partnership Project): a fully network-based Cell ID, a time-biased OTDOA-IPDL (observed time difference of arrival with idle period downlink), and AGPS [1].

\subsection{Enhancements to Cell ID}

A wide range of enhancements for the basic Cell ID technique have been developed mainly by utilizing standardized UE (user equipment) or UTRA (universal terrestrial radio access) physical layer measurements [11]. These enhancements mainly include Cell ID+RSCP (received signal code power) [12] and Cell ID+RTT (round trip time) that emerged from Cell ID+TA (timing advance) developed for GSM (global system for mobile communication) [13, 14]. Due to larger bandwidth and relatively short chip duration in UMTS $(0.26 \mu \mathrm{s})$, the accuracy of RTT measurements is significantly higher than the resolution of the corresponding TA-based technique in GSM $(\sim 550 \mathrm{~m})$. Theoretically, based on a single RTT measurement, mobile-to-base station distance can be estimated with an accuracy of $36 \mathrm{~m}$ with $1 / 2$ over sampling or, for instance, with an accuracy of $5 \mathrm{~m}$ when $1 / 16$ over sampling is applied at the base station. However, in practical implementation, the accuracy of estimates is reduced by multipath propagation and by application of receiver structures that do not feature high-order oversampling schemes. Typically, the overall accuracy of the Cell ID+RTT is expected to be at a greater level in the microcellular environment, as the probability of an LOS (line-of-sight) connection with the base station is higher. Moreover, range of positioning error is minimized in denser cell deployment. The performance of the Cell ID+RTT is comprehensively assessed in [15], as well as in the following sections of this paper.

\subsection{OTDOA- and AOA-based techniques}

In addition to the Cell ID, enhancements to the OTDOA technique have also been considered. The accurate OTDOA positioning requires simultaneous availability of three pilots from different sites, which is limited in typical UMTS scenarios. Hence, enhancements to the OTDOA technique are mainly focused on improving hearability of a distant pilot during positioning measurements. Standardized IPDL scheme involves synchronously ceasing transmission 
of the base station in order to maximize the hearability of distant pilots during the positioning measurements. Proposed enhancements consist of TA-IPDL (time alignedIPDL) [16, 17], PE-IPDL (positioning elements-IPDL) [18], and software-based technique called CVB (cumulative virtual blanking) [19]. TA-IPDL defines a specific, time-aligned configuration of IPDL periods from the different base stations. Namely, each involved base station is obligated to transmit the pilot for $30 \%$ of time and for the remaining time to cease its transmission allowing more distance base stations to be hearable by the UE. In turn, the PE-IPDL technique exploits additional network elements, which in a synchronized manner transmit DL (downlink) sequences that the UE can utilize to complement standardized OTDOA measurements. Hence, the hearability of signals from different transmitters is significantly improved by cost of the overall complexity increase. Alternatively to the IPDL-based techniques, availability of distant base stations can be maximized by exploitation of signal processing techniques that reduce unwanted interference as proposed in the CVB method. The accuracy provided by the depicted OTDOA-based techniques is maintained at the sufficient level for most of the LBS. For example, the TA-IPDL provides position estimation with $30 \mathrm{~m}-$ $100 \mathrm{~m}$ accuracy for $67 \%$ of measurements in urban environment $[16,17]$. Similarly, exploitation of the PE-IPDL technique can improve the attainable positioning accuracy by almost $15 \%$ (strictly depending on the number of used PEs) in heavy urban environment in comparison with the standardized OTDOA-IPDL [18]. Application of the software-based CVB method improves the hearability of distant pilots required for the OTDOA measurements that in turn narrows the possible location error to $12 \mathrm{~m}-24 \mathrm{~m}$ for $67 \%$ of estimates [19]. However, as a UMTS network is not synchronized, the combination of three SFN-SFN (system frame number) measurements, which constitutes the basis for all OTDOA-based techniques, requires utilization of LMUs providing real-time difference between involved NodeBs and the UE. Alternatively, the reliability of the OTDOA measurements in an unsynchronized network can be ensured by deployment of the PEs [18]. Due to LMU implementation costs, the applicability of the OTDOA-based techniques is problematic, especially when the AGPS-based positioning constitutes the longterm deployment objective. Implementation costs are estimated at the level of $8000 €$ per LMU together with annual maintenance costs at the level of $20 \%$ of the unit cost [20]. Depending on the density of the topology, one LMU can serve from 1 to 5 sites.

Other positioning techniques have also been proposed, for example, Matrix [21], which does not require implementation of LMUs to provide timing information, but exploits an exchange of data between users in the service coverage. This method utilizes measurements of relative timings of network signals received by the UE for derivation and maintenance of network synchronization map that in turn allows for position estimation based on time measurements. Matrix provides accuracy at a level of $50 \mathrm{~m}-90 \mathrm{~m}$ for $67 \%$ of measurements, but at the same time the method requires modifications at two communication ends.
Significant attention has also been gained by positioning methods utilizing AOA (angle-of-arrival) information of the UL (uplink) signal at the NodeB antenna $[22,23]$. The $67 \%$ CERP (circular error probability) of the AOA estimation is not expected to exceed $250 \mathrm{~m}$ in considered urban propagation environments. Furthermore, lots of hybrid approaches involving the AOA measurements have been proposed. For example, a conjunction of the UL TOA (time-ofarrival) information with the AOA slightly improves the accuracy $[24,25]$. Significantly, larger improvement has been reported in $[26,27]$, where the OTDOA measurements performed by the UE support the AOA measurements at the base station. This hybrid approach has revealed the accuracy at the level below $100 \mathrm{~m}$ for $67 \%$ of location estimates in most of the simulated configurations for urban environments. However, as the implementation of the AOA recognition technology requires utilization of adaptive array antennas, the applicability in current UMTS deployments is at the very low level.

\subsection{Database techniques}

Numerous proposed approaches to the positioning intended for urban environments are based on a database consisting of the most expected reports in the defined area. Simply, based on a priori knowledge of a particular measurement in the entire network, the position of the UE can be estimated in the region corresponding to the sample characterized by the highest degree of correlation with the actual measurement. For GSM, a method utilizing database with premeasured signal strength samples has been proposed in [28] and further intensively evaluated, for example, in [29]. Samples required for creation of the database can be collected by conducting measurements over the service area, but logically they can also be gathered by performing simulations, as presented in [30]. Reported accuracy has not exceeded $80 \mathrm{~m}$ for $67 \%$ of measurements. In turn, for UMTS networks, the DCM (database correlation method) has been developed [31]. This technique uses measurements of multipath delay profile from the strongest cell. Moreover, the complementary use of RTT information from the base stations improves the accuracy. The simulation results have shown that in very dense network scenarios for urban deployment, $67 \%$ of users can be located with an error smaller than $25 \mathrm{~m}$. In comparison, standardized OTDOA positioning evaluated in the same environment provided accuracy at the level of $97 \mathrm{~m}$ for $67 \%$ of measurements [31]. However, the short-term implementation constraint constitutes a fact that the UE impulse response measurements are not standardized, and thus deployment of the DCM requires changes in the standard terminals. Moreover, reporting of such measurements to the location server is also not specified in the 3GPP. Therefore, the applicability of the DCM is not at a high level in the current competitive market.

\subsection{Satellite-based techniques}

In addition to the development of cellular location methods, satellite-based solutions have also progressed in recent years. 
There are numerous developed commercial AGPS solutions for UMTS, for instance, gpsOne by Snaptrack (a Qualcomm company) [32] or IndoorGPS by Global locate [33]. Moreover, there is a concept actively studied within 3GPP work groups that utilizes navigation data of future positioning system-GALILEO. Namely, two approaches are considered: a method exploiting cellular assistance-assisted GALILEO and a method that utilizes both GPS and GALILEO data (AGPS + assisted GALILEO) for mobile positioning in UMTS [34].

\section{PROPOSED NETWORK-BASED LOCATION TECHNIQUES}

\subsection{Enhanced Cell ID+RTT}

The enhanced Cell ID+RTT method constitutes the hybrid extension to the basic network-based standardized positioning technique utilizing Cell ID information of the serving sector. The accuracy of the Cell ID can be improved by incorporation of a single RTT [11] measurement performed on the DPCH (dedicated physical channel) that is established in the Cell_DCH state. However, as presented in [15], the overall accuracy is not at a sufficient level for current LBS requirements. During SHO (soft handover), the presence of multiple dedicated connections can easily be exploited for combining RTT information measured by all NodeBs in the AS (active set), thus improving the overall Cell ID+RTT accuracy. According to regular SHO procedure [35], the radio link is added to the AS when the measured $E_{c} / N_{0}$ (energy per chip over interference spectral density) of the $\mathrm{CPICH}$ (common pilot channel) from the monitored cell is larger than the $E_{c} / N_{0}$ of the best server diminished by the adding range. Similarly, the cell is removed from the AS if the power of its pilot drops below $E_{c} / N_{0}$ of the best server minus the dropping range. However, the actual implementation of the SHO algorithm is vendor-specific. Earlier studies have shown that even highly overlapped topologies for urban UMTS deployment, for example, 6-sectored configuration with horizontally wide $\left(65^{\circ}\right)$ antennas, only provide up to $40 \%$ of SHO [15]. Thus, the overall accuracy of the traditional Cell ID+RTT is not at the sufficient level. Moreover, deployment of wide beamwidth antennas reduces the system capacity in a majority of topologies, since as presented in [36] utilization of horizontally narrow $\left(33^{\circ}\right)$ antennas can provide up to $40 \%$ capacity gain with respect to configuration with $65^{\circ}$ antenna beamwidth. In turn, widening the $\mathrm{SHO}$ window globally for the whole network will significantly reduce the DL capacity. Alternatively, if only the located UE is forced to SHO for a time instant needed to perform RTT measurements from the AS sites, the resulting increase of interference is not expected to affect the network capacity significantly. In locations near the serving NodeB, the accuracy of a single Cell ID+RTT is already at a good level, and moreover the probability of LOS measurement is high. Thus, the UE is forced to SHO only when reported single RTT corresponds to the distance that exceeds $150 \mathrm{~m}$. For instance, the accuracy of the single Cell ID+RTT at a distance of $150 \mathrm{~m}$ from the serving
NodeBs corresponds to $99 \mathrm{~m}$ and $57 \mathrm{~m}$ (6-sectored $/ 65^{\circ}$ scenario), and to $95 \mathrm{~m}$ and $16 \mathrm{~m}$ (6-sectored $/ 33^{\circ}$ scenario) for a single sector ID and softer handover area, respectively, when LOS is assumed [15].

The FSHO (forced SHO) procedure is triggered by an appropriate Measurement Control message [2]. The algorithm widens the $\mathrm{SHO}$ window by increasing the adding range for the particular UE until three pilots from different sites fulfill the adding criteria, that is, until corresponding $E_{c} / N_{0}$ measurements exceed the adding threshold (Figure 1). At the same time, the dropping range is adequately increased in order to prevent losing the added radio link before RTT measurements are successfully conducted. In locations in which three pilots are not simultaneously hearable, the algorithm exits after reaching the defined maximum allowed value for the adding range. Then, the UE sends Event $A$ message to the SRNC (Serving Radio Network Controller) in an adequate Measurement Report that triggers the AS update procedure [35]. Subsequently, all NodeBs included in the AS measure the RTT and report to the corresponding SRNC. Additionally, the reliability of the positioning in a multipath propagation environment can be improved by requesting multiple RTT measurements from a single link. Obtained reports are thereafter transmitted to the SMLC (Serving Mobile Location Centre), where they are further processed. Network is restored to the initial state by triggering a regular AS update procedure based on standardized measurements reported by the UE. The estimation of the position of the UE is performed by a constrained LS (least-square) numerical approach, because the error in the range estimation due to multipath propagation is always positive (LS technique is introduced in Section 5). Next, the estimated position of the UE is checked to which sector ID area it geometrically belongs. Under circumstances that the sector ID which corresponds to the estimated position of the UE does not match with the real sector ID of the UE, the accuracy can be enhanced by using the VM (virtual mapping) algorithm [3]. The VM procedure changes the estimated position to the nearest point that geometrically belongs to the area of the original sector ID of the UE. Implementation of the VM consists of a geometric definition of approximate cell dominance and SHO areas. In the case of uniformly distributed cells, deployment of the VM is not complicated. Distribution of cell dominance areas and SHO regions over the planned service area can be directly obtained, for instance, from the coverage predictions of the network plan. Naturally, with irregular network topology, implementation of the VM is becoming more complicated.

\subsection{Pilot correlation method}

The PCM is an entirely network-based approach and it does not require any hardware or software modifications in the UE [4]. This technique uses a database deployed in the network, which consists of the most probable view of $\mathrm{CPICH}$ levels for each defined positioning region. Positioning region is the selected area within the network coverage, for which an individual entry in the database is related. Positioning regions can be defined freely according to the requirements of 


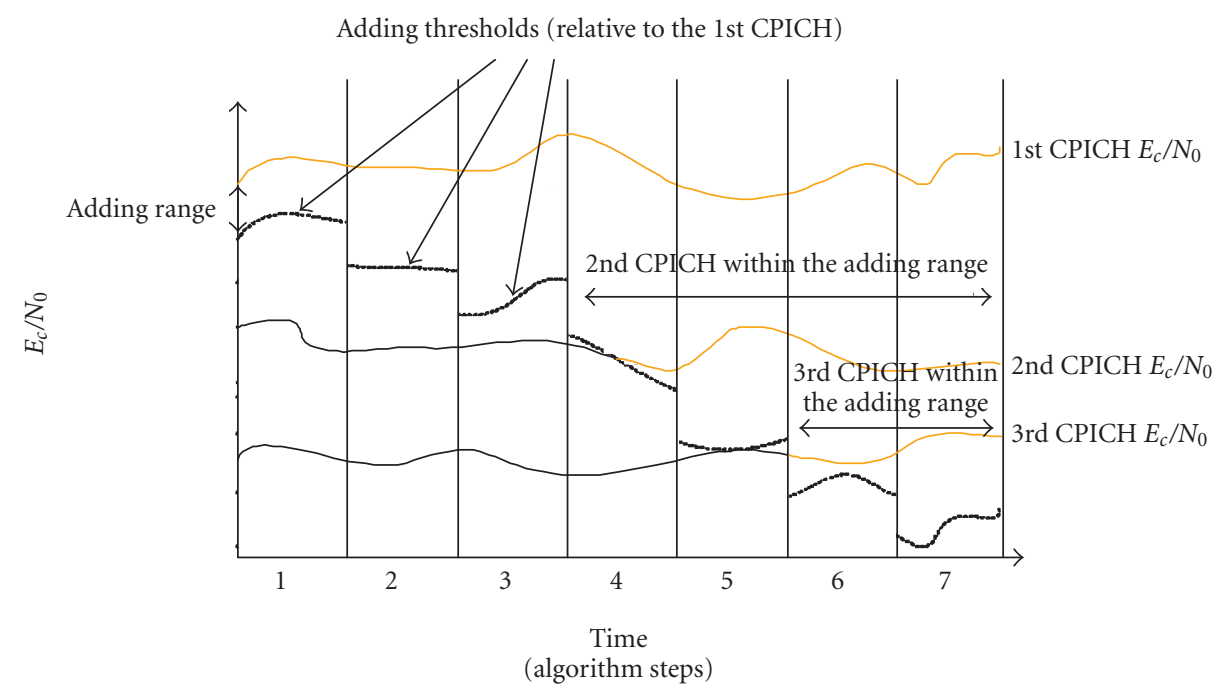

(a)

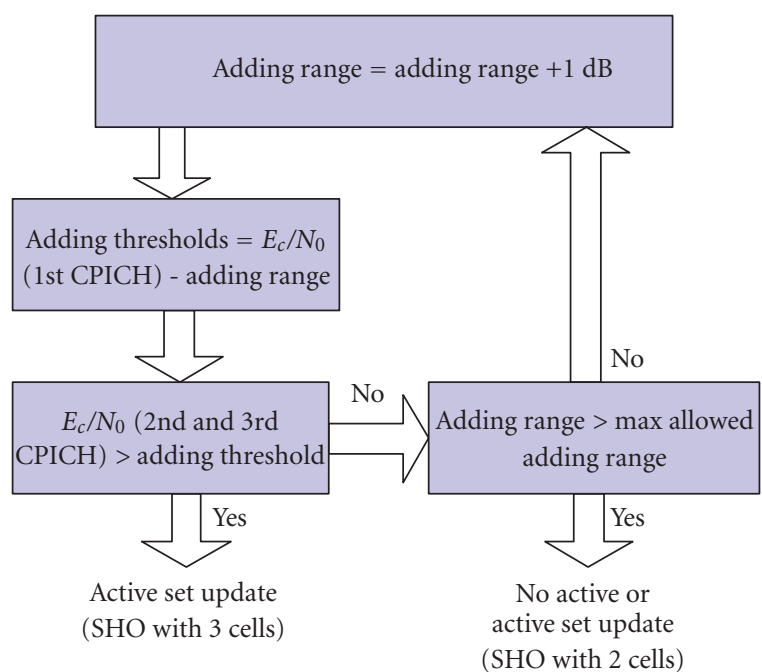

(b)

FIGURE 1: (a) Illustration of adding range in consecutive steps of the FSHO (forced SHO) algorithm execution; (b) simplified flow of the FSHO procedure.

planned LBS applications. Naturally, the size of the positioning region determines the resolution of the estimation and thus it limits the attainable accuracy of the PCM.

During regular network operation, when the UE is in the Cell_DCH or Cell_FACH state, the required information is continuously updated in the SRNC. Depending on the network configuration, the UE internal measurements are reported either periodically or they are triggered by variations of pilot levels. Therefore, in most of the situations, the information required for position estimation is already in the network. When the Location Request of the particular UE is received by the SRNC/SMLC, the latest valid measurement reported by the UE is selected and transferred to the SMLC for calculation of correlation with the stored samples in the database. If the most recent Measurement Report in the SRNC has been received a relatively long time ago, the information needs to be updated by executing a paging procedure in order to receive the latest Measurement Report message from the UE. Actual definition of expiration of measurements reported by the UE depends on the intended positioning accuracy and expected maximum velocity of the terminals in the considered network environment. For instance, for an urban scenario in which the velocity of majority of terminals does not exceed $40 \mathrm{~km} / \mathrm{h}$, definition of $5 \mathrm{~s}$ expiration time of reported measurement allows for keeping the accuracy within $100 \mathrm{~m}$. When the located terminal is in the other RRC (radio resource control) state in which the UE measurements are not reported, the paging procedure also needs to be performed. The SRNC pages the UE in order to cause a transition to the Cell_FACH state for a time instant that is required to receive the message containing the RSCP measurements of the pilots. Therefore, the method can be applied to regular 


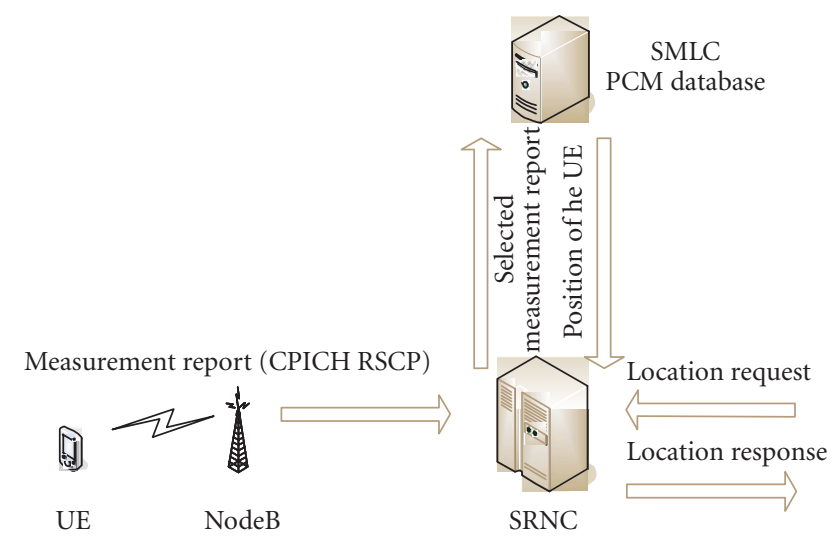

FIgURe 2: Pilot correlation method functional procedure.

terminals for UMTS, as the whole interaction with the UE is based on the standardized messages. The simplified flow of the PCM is presented in Figure 2. Naturally, the indicated Location Request can be initiated by the UE as well. When the selected Measurement Report is forwarded to the SMLC, the corresponding vector containing scrambling code IDs and measured RSCP of visible pilots is compared with the stored samples in the database. The location of the UE is estimated in the positioning region that corresponds to the sample that has the highest correlation with the measurement. Correlation is computed using the LS method, which is described in Section 5. In order to decrease the duration of the correlation process with the stored samples, the database is divided into parts depending on the scrambling code ID of the first pilot. Next, the measured sample is compared only with the stored samples, which are identified by the same scrambling code ID of the first pilot. Moreover, if there is a high probability of an erroneous assignment of the UE position to the positioning region (e.g., due to definition of very small positioning regions), it is beneficial to verify whether the correlation degree fulfills a defined threshold. If the threshold is not reached, a vector with RSCP data is formed from the average of the multiple latest RSCP measurements provided in the Measurement Reports to the SRNC. The position of the user is always estimated in the middle point of the positioning region, thus the error is minimized.

Creation of the database is an automatic process, as the implemented software generates a database from the log files of the radio interface measurement tool. Due to the crucial requirements of performing intensive field measurements during radio network planning and optimization phase, creation of the database does not involve extra effort. Logically, the database can also be generated from predicted values by a radio network planning tool. Under regular operation of the positioning method, the database should be updated from time to time (e.g., once in 6-12 months) due to propagation changes caused by modification of the urban scenario. Moreover, the database has to be updated as well if the network configuration is changed. The error of the estimation may rise for positioning regions located at the cell edge, since for these areas the probability of having a similar situation of visible pilots can be relatively high. However, the database can easily be complemented by exploiting GSM signal level experienced by the UE. Thus, the estimation accuracy can be further improved. In the situations where the degree of correlation is below the defined threshold, the SRNC can request intersystem measurements from the UE and perform the recorrelation process based on the obtained additional information. In a similar manner, the accuracy of the database can be enhanced by utilization of the most expected RTT data for each positioning region.

\section{ESTIMATION METHODS}

Proposed cellular positioning techniques require utilization of numerical mechanisms for minimization of the positioning error. The ECID+RTT method utilizes constrained LS (least-square) optimization for estimating the position from obtained distances to the NodeBs. In turn, the PCM exploits the LS method for calculating a deviation between the measurement and the samples stored in the database.

\subsection{Enhanced Cell ID+RTT}

Phenomena in the air interface, for example, multipath propagation, cause errors in measurements of cellular positioning techniques. Hence, a position estimation procedure from the reported ranges requires application of numerical approaches. Estimation of ranges that is performed by a timebiased cellular positioning method always consists of a positive error, thus the position of the UE can be derived by applying a constrained LS approach [37]. This algorithm assumes awareness of the rough position of the UE $(x, y)$, immobility of the UE during the positioning procedure, and omission of the third dimension (altitude). Typically, the initial position of the UE needed for the first iteration is assumed to constitute a center of gravity, which is indicated by the locations of neighboring NodeBs. Based on the stated assumptions, a positioning problem can be solved by processing at least two measurements expressing distances to different NodeBs. The position is estimated by minimizing a function $F(\mathbf{x})$ :

$$
F(\mathbf{x})=\sum_{i=1}^{N} f_{i}^{2}(\mathbf{x})-P \sum_{i=1}^{N}\left[\frac{1}{g_{i}(\mathbf{x})}\right]^{-1},
$$

where $\mathbf{x}$ stands for a single column matrix consisting of the coordinates of the UE $(x, y)$, and function $P$ is always a positive scalar. Moreover, $g_{i}(\mathbf{x})$ represents a penalty function defined as $g_{i}(\mathbf{x})=-f_{i}(\mathbf{x})$, and $f_{i}(\mathbf{x})$ is a function constituting a performance measure in respect to the $i$ th NodeB, as expressed in (2). The penalty function is introduced in order to form an applicable solution by employing an unconstrained LS optimization method, that is, when the introduced error has an undefined sign. This approach allows for relatively fast convergence without usage of high computation power:

$$
f_{i}(\mathbf{x})=d_{i}-\sqrt{\left(x_{i}-x\right)^{2}+\left(y_{i}-y\right)^{2}} \geq 0 .
$$


In (2), $d_{i}$ is the measured range defined by RTT measurement from the $i$ th NodeB. Moreover, $x_{i}$ and $y_{i}$ represent the coordinates of the $i$ th NodeB. The function $f_{i}(\mathbf{x})$ is always positive as the real position of the UE is always within the area constrained by boundaries, which are defined by estimated cellular ranges. Successive location estimates are updated according to the following recursion:

$$
\mathbf{x}_{k+1}=\mathbf{x}_{k}-\mu \nabla_{x} F\left(\mathbf{x}_{k}\right) .
$$

The parameter $\mu$ represents the recursion step (scalar or diagonal matrix) and $\mathbf{x}_{k}$ is a single column matrix consisting of the UE coordinates $\left(x_{k}, y_{k}\right)$. The minimization is continued until condition (4) is fulfilled for a defined threshold $(t)$ :

$$
\left\|\nabla_{x} F\left(\mathbf{x}_{k}\right)\right\| \leq t
$$

For the first iteration, $P$ is selected to be reasonably large. After reaching the convergence stated in (4), the minimization procedure given by (3) is repeated with smaller value of $P$ (such as $P_{n+1}<P_{n}$ ), and the previous estimate $\left(\mathbf{x}_{k}\right)$ is used for the first iteration. The approach is continued as long as subsequent iterations introduce change in the final estimate $\mathbf{x}_{k}$ in the order of $10 \mathrm{~m}$ or more.

In addition to the constrained LS method, there are other approaches applicable for solving the position from the range information, for example, a method which is based on Taylor linearization [38].

\subsection{Pilot correlation method}

An uncomplicated LS approach is used to compute the deviation $\left(S_{\mathrm{LMS}}\right)$ between the stored samples in the database, and the actual reported measurement:

$$
S_{\mathrm{LMS}}=\sum_{i \in N}\left(s_{i}-m_{i}\right)^{2}=\sum_{i \in N} \Delta_{i},
$$

where vectors representing the stored sample and the reported measurement are indicated by $s_{i}$ and $m_{i}$, correspondingly. This deviation is computed for all fields included in the vector $(N)$ and it is applied for all samples stored in the relevant part of the database according to the particular scrambling code ID. The UE is estimated in the positioning region corresponding to the sample, which is characterized by the minimum deviation.

\section{SIMULATION ENVIRONMENT AND MEASUREMENT SCENARIO}

Different approaches were taken for performance evaluation of the proposed positioning techniques. Namely, the performance of the ECID+RTT was assessed by extensive simulations in various topology and environmental configurations whereas the applicability of the PCM positioning was verified by conducting measurement campaigns in an urban and suburban UMTS network. Moreover, impact of the FSHO procedure on UMTS network capacity was evaluated by measurements in an indoor UMTS network.

\subsection{Enhanced Cell ID+RTT}

A Matlab-based simulator was implemented for the performance examination of the ECID+RTT under various propagation conditions. A network layout used for simulations consisted of equally spaced ( $1 \mathrm{~km}$ ) 6-sectored sites in a hexagonal grid with constant antenna directions. Mobiles were randomly distributed over the simulation area. In the performed simulations, continuous availability of the FSHO was assumed. For a randomly selected mobile, RTT measurements from three sites were simulated. Two different propagation environments (urban and suburban) were considered with different expected errors in RTT measurements. The effect of NLOS (non-LOS) on range measurements was modelled by a positive, distance-dependent error, such as $i$ th measured RTT was defined as

$$
\operatorname{RTT}^{i}(d)=L^{i}(d)+2 \cdot \operatorname{NLOS}^{i}(d) .
$$

In (6), $L^{i}(d)$ is the RTT that corresponds to the LOS measurement from the $i$ th base station, and $d$ represents the distance from the mobile to the base station. Since RTT measurement suffers from NLOS bias in both directions (DL and $\mathrm{UL})$, the additive error is doubled. The positive NLOS bias was approximated by the mean excess delay $\left(\tau_{m}\right)$ of the radio channel based on the studies presented in [39].

Moreover, according to wideband channel measurements cited in [39], the mean excess delay is essentially correlated with the root-mean-squared delay spread ( $\left.\tau_{\mathrm{RMS}}\right)$ of the channel:

$$
\operatorname{NLOS}^{i}(d) \approx \tau_{m}^{i} \approx k \cdot \tau_{\mathrm{RMS}}^{i}
$$

The scaling factor $k$ was derived to be approximately 1 for urban and 2 for suburban environment. The expected value of $\tau_{\mathrm{RMS}}$ in a function of mobile-to-base station distance can be estimated based on the model presented in [40]. The referred statistical model defines that the median $\tau_{\mathrm{RMS}}$ increases with $d^{\varepsilon}$, where an exponent $\varepsilon$ equals 0.5 for urban and suburban propagation environments. According to (7) and the distance-dependent delay spread model, the value of the additive NLOS bias can be approximated by the following equation:

$$
\operatorname{NLOS}^{i}(d) \approx k \cdot \tau_{\mathrm{RMS}}^{i}(d) \approx k \cdot T_{1}\left(d^{i}\right)^{\varepsilon} \cdot x^{i}
$$

In (8), $T_{1}$ stands for the median value of $\tau_{\mathrm{RMS}}$ at $d=1 \mathrm{~km}$ and $x^{i}$ is a $\operatorname{lognormal}$ variable, such as $X=10 \log (x)$ is a Gaussian-distributed random variable over the terrain at distance $d$ with zero mean and standard deviation $\sigma_{x}$. Reported measurements in $[41,42]$ provide mean $\tau_{\mathrm{RMS}}$ observed at the distance of $1 \mathrm{~km}$ from the base station, namely, $T_{1}=0.92 \mu \mathrm{s}$ and $0.27 \mu \mathrm{s}$ for considered urban and suburban environments, correspondingly. For considered environments, standard deviation $\left(\sigma_{x}\right)$ was assumed to be $2 \mathrm{~dB}$ for suburban and $4 \mathrm{~dB}$ for urban scenario [40]. Since $\operatorname{NLOS}^{i}(d)$ is always positive, negative samples of random variable $x^{i}$ were omitted. An example of the modelled range errors is illustrated in Figure 3. 


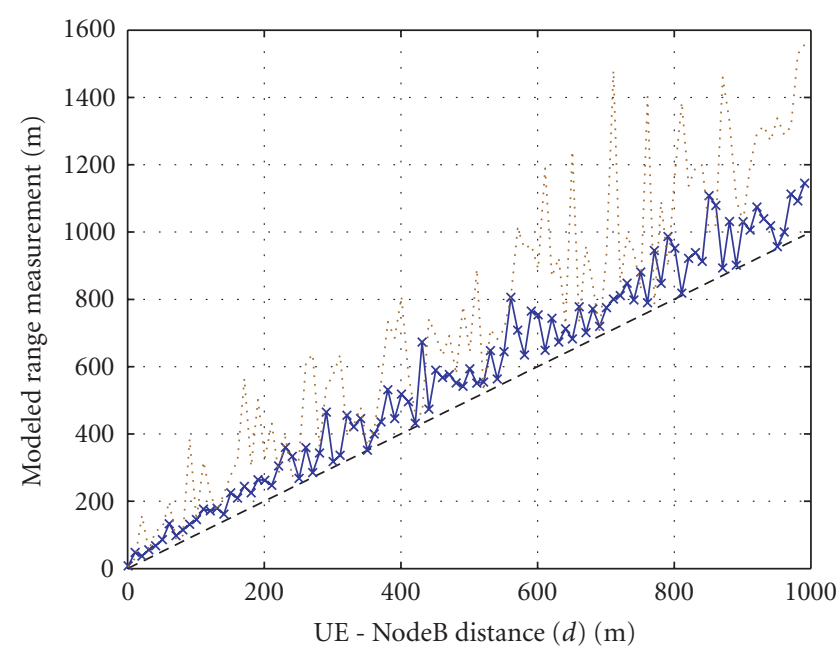

- - - Modeled erroneous range in urban environment

$\rightarrow$ Modeled erroneous range in suburban environment Line-of-sight distance

Figure 3: Modeled range error for considered multipath models in a function of the UE-NodeB distance.

TABLe 1: Probability of multipath model selection in the second iteration depending on the simulated propagation environment.

\begin{tabular}{l|cc}
\hline Propagation environment & \multicolumn{2}{|c}{ Multipath model } \\
& Urban & Suburban \\
\hline Urban & 0.85 & 0.15 \\
Suburban & 0.1 & 0.9 \\
\hline
\end{tabular}

Subsequent iterations of range measurements on each link were performed for reliability improvement in multipath propagation environments. Logically, on each measured link, the smallest reported RTT was remembered for further position calculations. Each repetition of the RTT measurements in a certain propagation environment gives a small probability of defining the additive RTT error according to the model with parameters defined for different propagation environment. Weights for the model selection were determined in such a manner that the probability of selecting a model describing a different propagation environment than in the previous round was maintained at a low level (Tables 1 and 2). Simulations were performed for 4 and 10 RTT measurements on a single link. Obtained ranges were processed by the constrained LS optimisation. The position of the UE was estimated based on 30 iterations of the numerical procedure. The VM algorithm was utilized and assessed for 6sectored configuration with $65^{\circ}$ and $33^{\circ}$ antennas. The presented results of the accuracy constitute an average of 5000 location estimation processes in each simulated configuration.

The impact of forcing the UE to SHO on the network capacity was assessed by measurements performed in an indoor UMTS network. In the considered, four-storey building, cellular coverage was provided by DAS (distributed antenna
TABle 2: Probability of multipath model selection in the consecutive iterations depending on the multipath model selected in the previous iteration; LOS (line of sight).

\begin{tabular}{l|ccc}
\hline Multipath model in & \multicolumn{3}{|c}{ Multipath model in the next iteration } \\
the previous iteration & Urban & Suburban & LOS \\
\hline Urban & 0.85 & 0.15 & 0 \\
Suburban & 0.15 & 0.8 & 0.05 \\
LOS & 0 & 0.2 & 0.8 \\
\hline
\end{tabular}

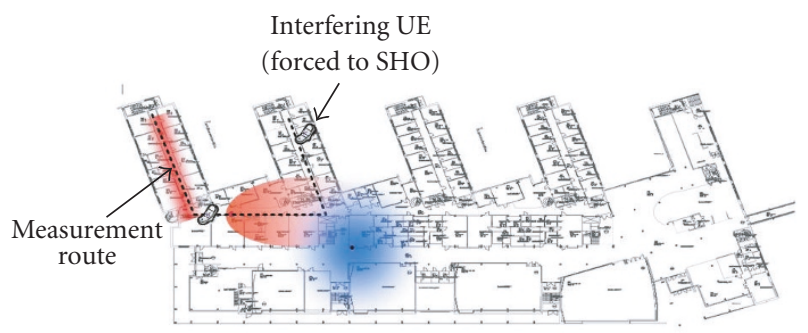

Figure 4: A part of the indoor network (cell 1: leaky feeder and discrete antenna, cell 2: omnidirectional antenna) with illustration of the measurement route and the location of the interfering UE.

system). The verification measurements were performed in the selected indoor area with two cells coverage provided by omnidirectional antenna, directional antenna, and leaky feeder (Figure 4). The network capacity in different FSHO situations was evaluated based on $E_{c} / N_{0}$ measurements collected over the defined route (Figure 4). The measurement equipment consisted of a laptop PC with UMTS radio interface measurement software connected to the test UE. Two FSHO situations were modelled by the UE that was forced to SHO in locations where the path losses to the hearable cells differed by $5 \mathrm{~dB}$ and $10 \mathrm{~dB}$. In the locations of the interfering $\mathrm{UE}$, the average $E_{c} / N_{0}$ of the dominant pilot was at the level of $-5 \mathrm{~dB}$. The interfering $\mathrm{UE}$ had a regular voice connection established. In order to minimize possible measurement error, statistics were gathered during 10 repetitions of the measurement route. Based on observed $E_{c} / N_{0}$ by the measured UE, the capacity loss was estimated according to the capacity evaluation method described in [43] and with the assumed frequency of arrival of positioning requests.

\subsection{Pilot correlation method}

Assessment of the applicability of the pilot correlation method was performed by measurement trials in an urban and suburban UMTS network. The first considered topology scenario was typical for dense urban deployment, as it consisted of 3-sectored sites with $400 \mathrm{~m}$ mean spacing distances. The average base station antenna height $(20 \mathrm{~m})$ slightly exceeded the rooftop level, thus forming a micro-/macrocellular scenario. In turn, the second network configuration constituted a typical macrocellular topology for suburban environment. Sites were $1.2 \mathrm{~km}$ distant from each other and 
the average base station antenna height was at an altitude of $25 \mathrm{~m}-30 \mathrm{~m}$, which was significantly higher than the mean rooftop level (residential area). Over 300 positioning regions were defined within selected areas of urban $\left(2 \mathrm{~km}^{2}\right)$ and suburban $\left(3.5 \mathrm{~km}^{2}\right)$ network coverage. In the urban network configuration, an average size of the positioning region and thus the minimum estimation region was roughly $100 \mathrm{~m} \times 50 \mathrm{~m}$. According to the smaller accuracy requirements of LBS for suburban areas, an average size of the positioning region in the second considered scenario was defined to be approximately $100 \mathrm{~m} \times 100 \mathrm{~m}$. Positioning regions were mainly selected in a manner that a part of the street along the same building (i.e., from one corner to another) corresponded to one positioning region. In areas with an irregular grid of streets and buildings, multiple positioning regions were defined within the same street or square in order to maintain the intended average size of the positioning region. RSCP samples required for the database creation were collected by a measurement tool consisting of the laptop PC with UMTS air interface measurement software connected to the test UE and the GPS receiver. Evaluation of the accuracy was performed by the user moving along two defined routes in each analyzed network environment. During each route, the position was estimated over 2000 times. The reported accuracy constituted a difference between the reported position and the indication of the GPS receiver.

\section{PERFORMANCE OF POSITIONING: RESULTS AND ANALYSIS}

\subsection{Enhanced Cell ID+RTT}

Figure 5 illustrates the reported accuracy of the ECID+RTT positioning in two considered propagation environments. In the simulated urban scenario, where the NLOS errors in RTT measurements are the largest, application of the VM can significantly increase the accuracy. For instance, in the 6 -sectored $/ 65^{\circ}$ scenario, the accuracy for $67 \%$ of location measurements equals $125 \mathrm{~m}$ without the VM and $100 \mathrm{~m}$, when the VM procedure is applied (Figure 5(c)). Expectedly, the overall accuracy is radically better with higher number of RTT iterations, since probability of more reliable RTT measurement is increased (Figure 5(d)). Simultaneously, in a configuration that performs 10 RTT measurements on each radio link, the application of the VM does not bring as significant an improvement as was observed with 4 iterations of RTT measurements. For instance, in the 6-sectored $/ 65^{\circ}$ topology evaluated in urban propagation environment with 10 consecutive RTT measurements from each NodeB, the accuracy for $67 \%$ of location estimates is at the level of $60 \mathrm{~m}$ and $65 \mathrm{~m}$ with and without the VM, correspondingly (Figure 5(d)). The accuracy of the ECID+RTT technique does not change much when it is deployed on top of different network topologies. As indicated in Figure 5, the positioning in the 6 -sectored $/ 65^{\circ}$ network topology has a slightly better accuracy than in the 6 -sectored $/ 33^{\circ}$ scenario. On average, the mean accuracy is improved by $5 \mathrm{~m}-10 \mathrm{~m}$ and the variance is improved by $5 \mathrm{~m}$ in comparison to deployment in the 6 -sectored $/ 33^{\circ}$ network. This fact is mainly caused by reduction of softer handover areas in the 6 -sectored $/ 33^{\circ}$ configuration, in which the accuracy is significantly better for mobiles located relatively near the serving $\operatorname{NodeB}(\leq 150 \mathrm{~m})$. Thus, mobiles in these areas are not forced to $\mathrm{SHO}$ as the single Cell ID+RTT accuracy is at the sufficient level. The accuracy of the ECID+RTT in environments with smaller expected multipath delays is naturally higher, as the 67\% CERP in 10 RTT iteration case decreases from $65 \mathrm{~m}$ in urban to $40 \mathrm{~m}$ in suburban environment, (Figures 5(b) and 5(d)). Latency of the whole positioning procedure is defined only by the duration of the FSHO algorithm, since fast convergence of the constrained LS method ( $<30$ iterations) together with the uncomplicated VM algorithm does not cause a noticeable delay. In turn, the duration of the FSHO procedure mainly depends on signaling delays. According to the latency analyses presented in [2] which were based on standardized maximum delay requirements $[44,45]$, total duration of the ECID+RTT positioning procedure does not surpass $2 \mathrm{~s}$.

\subsection{Pilot correlation method}

Figure 6 presents the cdf (cumulative distribution function) of the positioning accuracy reported by the PCM. Assessment of the accuracy in the micro-/macrocellular urban and macrocellular suburban environments is executed by locating the UE moving along two defined routes (indicated as solid and dashed lines in Figures 6(a) and 6(c)). Conducted measurements in the urban environment provide promising accuracy results (Figure 6(b)), since the accuracy for $67 \%$ of measurements is maintained below $70 \mathrm{~m}$. At the same time, the reported $90 \%$ CERP is from $130 \mathrm{~m}$ in case of the route 1 to $180 \mathrm{~m}$ in the case of the route 2 . The accuracy reported by the mobile travelling along the route 2 is evidently worse due to more locations close to the cell edge where the probability of erroneous estimation is higher, as pilots are hearable at similar levels in adjacent positioning regions. The achieved precision fulfils the defined FCC safety requirements for network-based solutions with a big margin and simultaneously it is sufficient for most of the location-sensitive applications. Similarly, in the case of the PCM operation in the typical macrocellular network, the accuracy is still maintained at a good level. However, due to larger site spacing distances and definition of larger sizes of positioning regions, the error is higher compared to the reported accuracy in the dense urban network. As indicated in Figure 6(d), the accuracy for $67 \%$ of measurements is reported at the level from $170 \mathrm{~m}$ to $190 \mathrm{~m}$. Since the resolution of the PCM positioning in the considered macrocellular topology is limited by characterization of the positioning region size $(100 \mathrm{~m} \times 100 \mathrm{~m})$, it is expected that for LBS requiring higher accuracy, the precision of estimation could be further improved by adequate definition of positioning regions.

The PCM exploits a single database that provides means for the positioning of multiple types of terminals, hence the accuracy of the method is directly sensitive to the accuracy of RSCP measurements performed and reported by the located UE. However, each Measurement Report that 


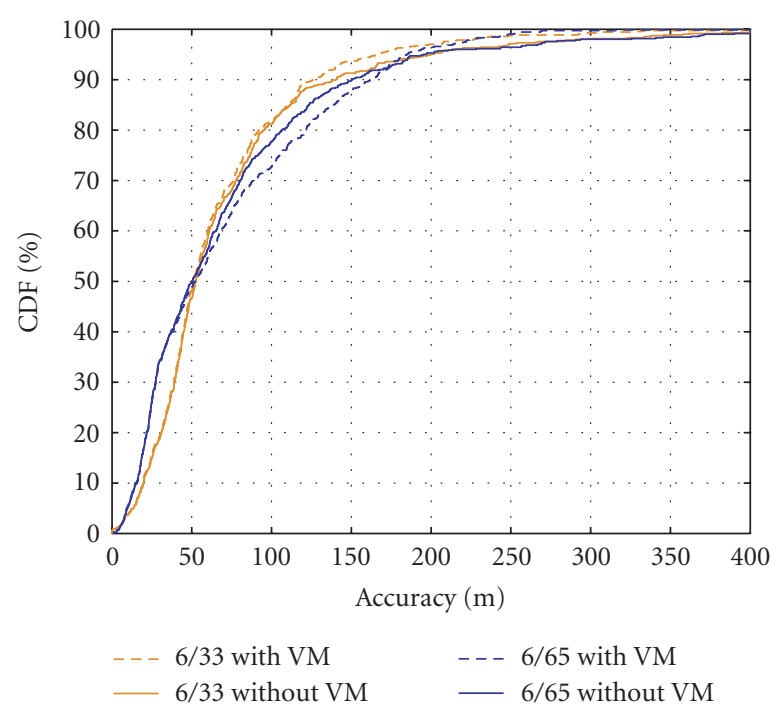

(a)

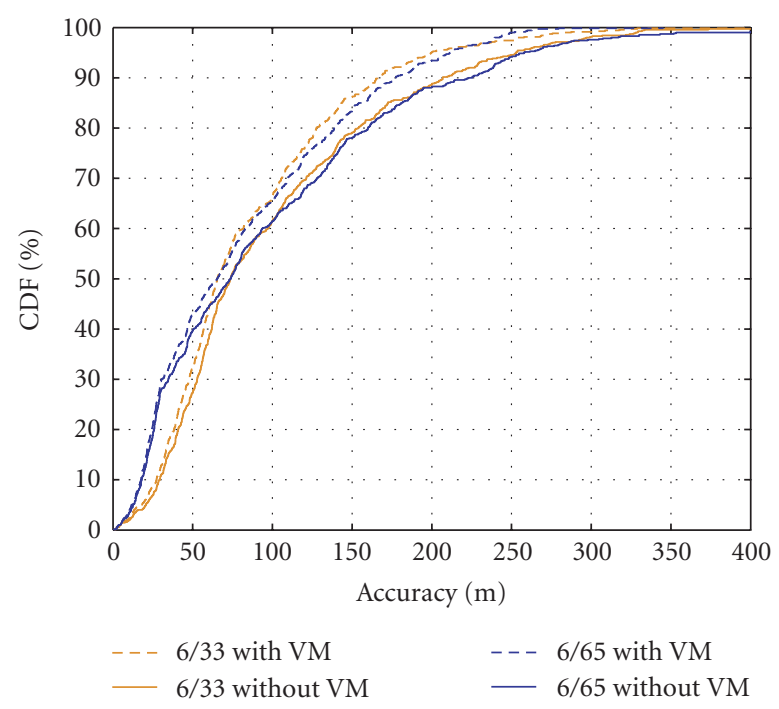

(c)

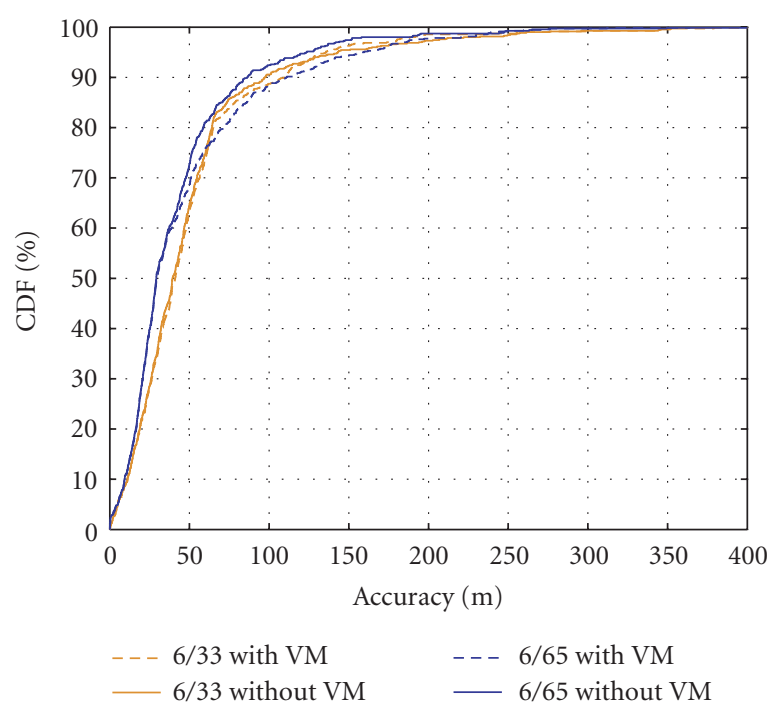

(b)

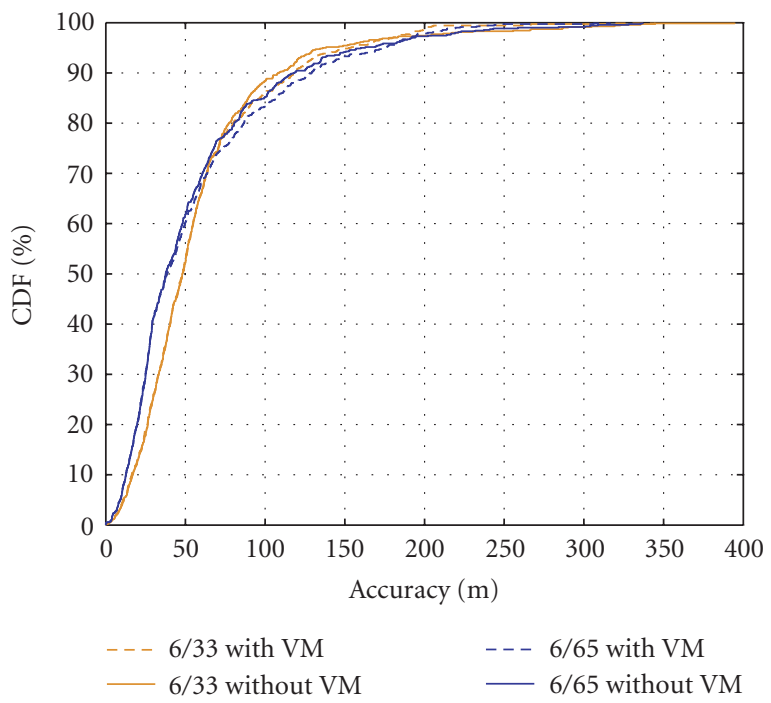

(d)

FIGURE 5: Accuracy results of the ECID+RTT positioning method for two different propagation environments and two iteration scenarios: (a) suburban with 4 iterations, (b) suburban with 10 iterations, (c) urban with 4 iterations, and (d) urban with 10 iterations.

is sent to the SRNC constitutes a mean value of multiple internal UE measurements. Thus, the deviations in accuracy of RSCP measurements in different terminals $( \pm 10 \mathrm{~dB})$ are averaged, minimizing the influence of the terminal type on the PCM performance. Naturally, performed averaging cannot entirely eliminate this measurement-specific uncertainty. Hence, slight deviations of positioning accuracy could occur for PCM estimation executed for different terminal types. Conducted field trials indicate that other factors contributing to the overall positioning performance (as latency and availability) do not have a limiting influence. Due to uncomplicated procedure, even if the update of Measurement Reports is needed, the latency is unnoticeable, as duration of the whole paging procedure should not exceed $0.4 \mathrm{~s}$ [45]. Also the availability does not limit the overall performance, because all served mobiles need to have the capability of reporting the measurements to the SRNC, from which the adequate RSCP values are extracted. Therefore, the PCM is available for all served terminals.

\section{IMPACT ON NETWORK PERFORMANCE: RESULTS AND ANALYSIS}

The ECID+RTT positioning technique can negatively affect the network performance especially when the UE is in a location that received power from monitored cells is at the minimum hearability level. Moreover, network capacity can be affected when the UE is forced to SHO in the location where the difference between received power levels from monitored 


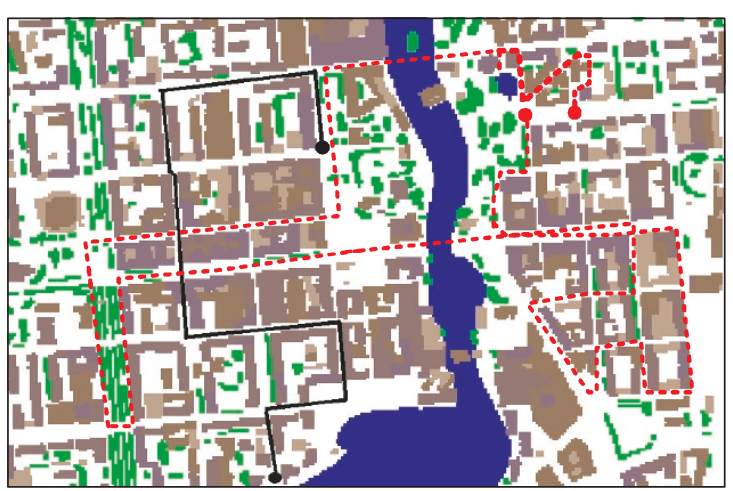

(a)

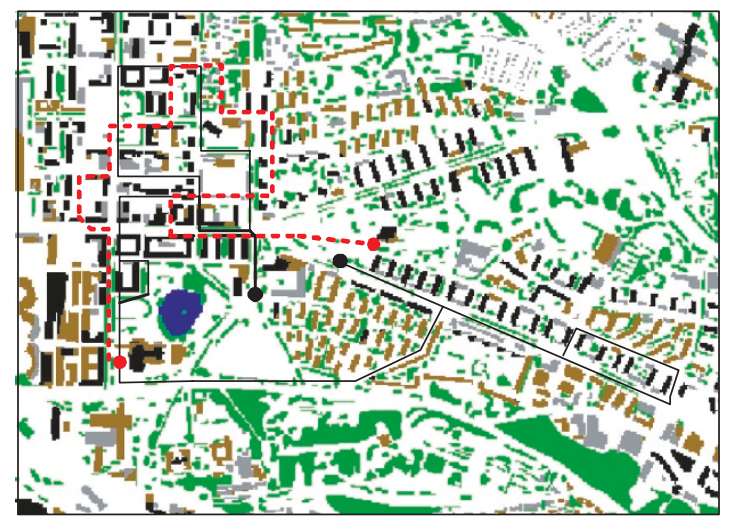

(c)

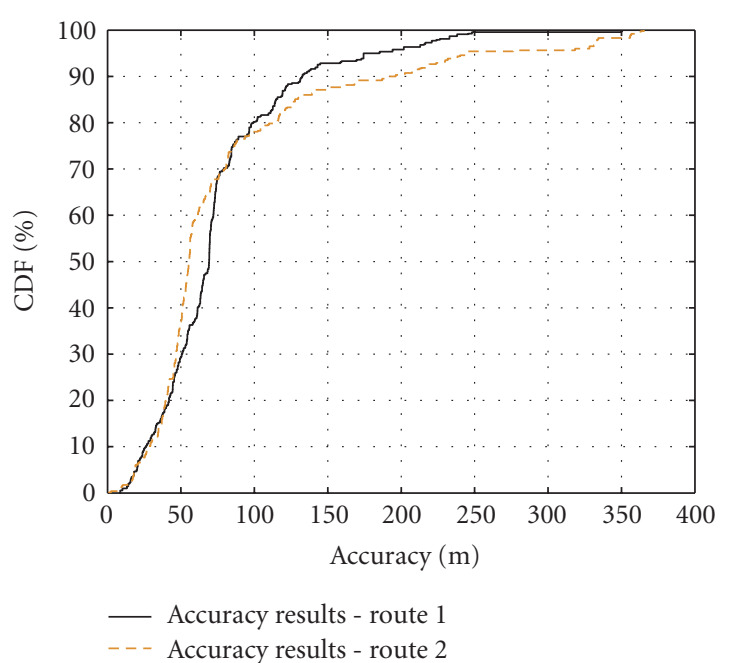

(b)

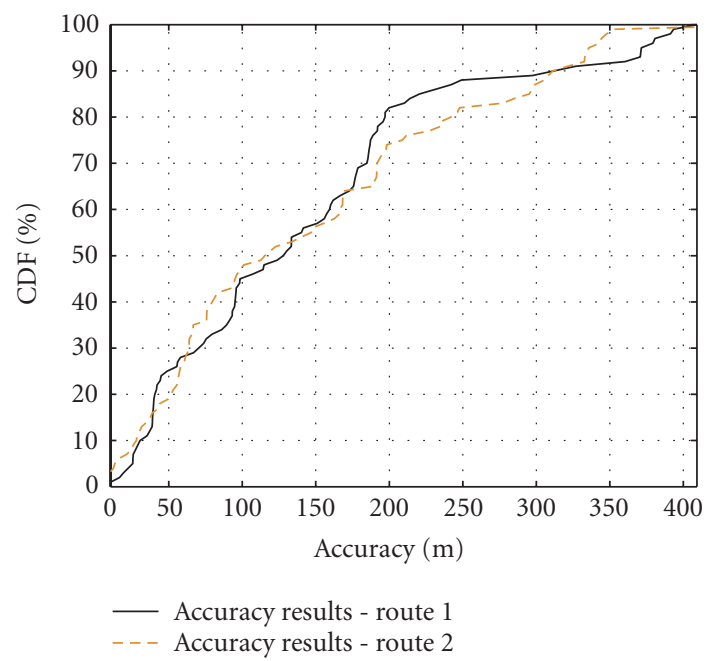

(d)

FIGURE 6: Measurement routes and accuracy results of the PCM positioning: (a) route in the urban micro-/macrocellular network, (b) corresponding accuracy results, (c) route in the suburban macrocellular network, (d) corresponding accuracy results; route 1 in both environments is indicated by the solid line, and route 2 by the dashed line.

cells is significant. Then, the radio links added through execution of the FSHO algorithm require excessive transmit power that in turn contributes to the higher interference level in the network. However, conducted measurements indicate that in practical scenarios, expected decrease of the network capacity is not large. $E_{c} / N_{0}$ is a ratio of RSCP of the first $\mathrm{CPICH}$ and RSSI (received signal strength indicator). Therefore, it provides a feasible reference for evaluation of the DL interference. If the interference level in the empty network is known, other-to-own cell interference $\left(i_{D L}\right)$ during execution of the FSHO procedure can be estimated according to measured $E_{c} / N_{0}(9)[43]$ :

$$
i_{D L}=\frac{\mathrm{RSCP} / I_{\mathrm{own}}}{E_{c} / N_{0}}-1 .
$$

The presented approach is applicable, since in an empty network the level of own cell interference $\left(I_{\text {own }}\right)$ can be easily captured by tracking $E_{c} / N_{0}$ measurements. Hence, increase of the $i_{D L}$ during operation of the FSHO can be directly obtained from decrease of measured $E_{c} / N_{0}$. The maximum achievable (average) DL capacity can be estimated by an inverse load curve assuming certain allowed noise rise; see [46] for load equations. The comprehensive description of the exploited capacity evaluation method is presented in [43]. In order to evaluate the capacity, in addition to the $i_{D L}$, the DL orthogonality factor $(\alpha)$ needs to be estimated. In performed analysis, the network capacity was evaluated based on a $3 \mathrm{~dB}$ allowed noise rise. Moreover, high orthogonality (0.9) of the analyzed indoor environment was assumed due to dominance of LOS connections within the considered route and due to the small time dispersion of the indoor channel. 
Performed measurements showed that when the interfering UE was in a regular state, that is, it was not forced to $\mathrm{SHO}$, the $i_{D L}$ was estimated at the level of 0.455 . During establishment of SHO, the $i_{D L}$ increased to 2.63 when the difference between measured $E_{c} / N_{0}$ from CPICH of AS cells was at the level of $5 \mathrm{~dB}$. In turn, when the path loss to the AS cells in the location of the interfering UE differed by $10 \mathrm{~dB}$, the $i_{D L}$ increased to 2.77 . The resulting capacity in the analyzed network without the FSHO operation is $1350 \mathrm{kbps}$. When the $\mathrm{UE}$ was forced to $\mathrm{SHO}$ with $5 \mathrm{~dB}$ and $10 \mathrm{~dB}$ differences between path losses to the AS cells, the instantaneous capacity dropped correspondingly to $220 \mathrm{kbps}$ and $200 \mathrm{kbps}$. A small difference in the observed capacity losses between the considered FSHO window scenarios can be explained by referring to the optimum SHO window. In indoor UMTS configurations, the optimum SHO window was observed to be relatively high, that is, approximately $7 \mathrm{~dB}$ [47]. Hence, with $5 \mathrm{~dB}$ difference between $E_{c} / N_{0}$ of the AS cells, the UE does not fully utilize the diversity and potential SHO gain is not effectively exploited. However, the $10 \mathrm{~dB}$ difference is already too large and the base stations have to transmit with excessive power to maintain the connection successfully, hence contributing to overall interference increase.

Indicated capacity losses illustrate only instantaneous capacity drops during the $\mathrm{SHO}$ establishment. In practical scenarios, the location requests are not expected to arrive continuously. For analysis, a uniform location requests arrival process was assumed. Moreover, three arrival rates were considered, that is, location request arrive every 30 seconds, 1 minute, or 5 minutes. The mean capacity of the cells involved in the FSHO procedure during an hour was estimated. Due to insignificant latency of the ECID+RTT positioning (2s), the higher level of interference during the existence of the additional radio links has negligible impact on the system capacity (Figure 7). The capacity of the cells that are involved in SHO drops by slightly more than $8 \%$ if location requests from a particular coverage area are received by the RNC every 30 seconds. Instantaneous capacity drops are not expected to be notable by majority of applications. Naturally, the highest peaks of interference would be introduced if the positioned UE was served by the cell that is clearly dominating. If multiple location requests are received by the SRNC simultaneously, a simple scheduling approach for the FSHO execution should be applied in order to prevent multiple UEs under the same cell coverage to be forced to SHO at the same time. Hence, potential negative consequences could be minimized. Indicated instantaneous capacity losses were observed for the UE that was forced to two-way SHO. However, the proposed procedure foresees three-way SHO if three pilots from different sites are receivable by the UE. Thus, it may be advantageous first to force the UE to two-way SHO to perform required RTT measurements. Then, release the added radio links and force the UE again to two-way SHO with different cell in order to acquire all needed measurements while avoiding significant instantaneous interference peak. Such a scheduling approach increases the latency by only $1 \mathrm{~s}$ and generates an insignificant signaling load. In addition, the presented scheduling approach for multiple location requests

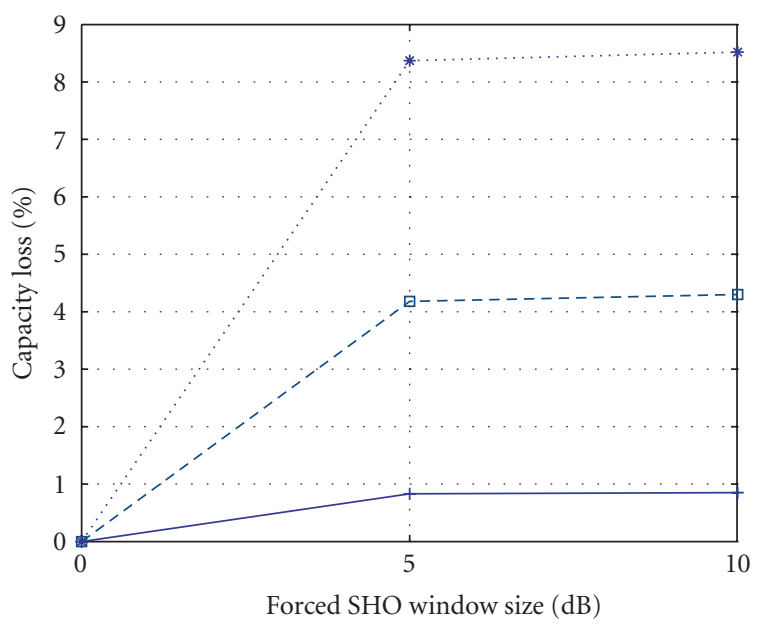

‥ Location request arrives every $30 \mathrm{~s}$
- - Location request arrives every $1 \mathrm{~min}$
+ Location request arrives every $5 \mathrm{~min}$

FIGURE 7: Expected capacity drop [\%] in cells involved in the FSHO procedure. Indicated loss depends on the pathloss difference to active set cells and the frequency of executing positioning procedure.

as well as avoidance of the establishment of three-way SHO minimizes a danger of reaching the maximum DL channelization code capacity under particular scrambling code.

The signaling load in the air interface needed for the operation of the FSHO procedure does not differ much from that of the traditional SHO operation, since only one extra pair of Measurement Control/Report messages is transmitted. Thus, the extended positioning procedure that includes consecutive establishment of two-way $\mathrm{SHO}$ requires additional exchange of only two pairs of messages. Furthermore, the additional radio links are not established when the UE is located very near the serving NodeB $(\leq 150 \mathrm{~m})$, since then the accuracy of a single RTT is already at the sufficient level. Hence, in most practical situations, the UE that observes significant $E_{c} / N_{0}$ difference between hearable CPICHs is not forced to $\mathrm{SHO}$ because most likely it is located sufficiently near the base station and the position can be estimated based on the single RTT measurement only.

\section{CONCLUSIONS AND DISCUSSION}

ECID+RTT and PCM network-based cellular positioning methods are proposed and evaluated. Complexity of the developed location methods is maintained at the minimum possible level in order to provide support for locationsensitive applications in current UMTS networks in a shortterm without requesting users to change their terminals. Correspondingly, the ECID+RTT technique requires only a slight software update in the user terminals in order to enable the FSHO procedure, while the PCM does not require any modifications in the terminals. The ECID+RTT method estimates the position of the terminal based on multiple time measurements. At the same time, the proposed method does not require implementation of LMUs for providing 
timing information that is usually mandatory with other time-biased location techniques. In addition, the ECID+RTT method requests numerous RTT from the same radio link and utilizes the proposed VM procedure that improves the positioning accuracy in the multipath environment. In turn, the PCM positioning is based on the database concept. The proposed method utilizes standardized measurements that are frequently reported by the user terminals and thus they are easily accessible in the network. Hence, the signalling overhead in the radio interface is minimized and the overall implementation process is straightforward.

The performance of the proposed methods is assessed using two different approaches: simulations and field measurements, mainly because the implementation of the ECID+RTT required for the measurement-based verification was not feasible in the considered UMTS network. In turn, the accuracy of the PCM positioning is relatively straightforward to assess by field measurements, as the impact of the proposed method on the regular network functionality is minor. Naturally, field measurements provide more reliable performance outcomes than simulations. Therefore, the applicability of the PCM is assessed by measurement campaigns performed in an urban and suburban UMTS network while the accuracy of the ECID+RTT is verified with simulations conducted in different topology and multipath propagation scenarios.

The reported accuracy of the ECID+RTT for $67 \%$ of the location estimates varies from $40 \mathrm{~m}$ in suburban to $65 \mathrm{~m}$ in urban environment when the VM procedure is used and the RTT is measured 10 times on each link. With a smaller number of RTT iterations, the accuracy of the ECID+RTT is still at a sufficient level for the majority of LBS, as the 67\% CERP is at a level of $70 \mathrm{~m}$ in suburban and $100 \mathrm{~m}$ in urban environment. The performed measurement campaign of the PCM provides positive results, as the accuracy for $67 \%$ of the measurements does not exceed $70 \mathrm{~m}$ in the dense network topology for urban environment. Similarly, field measurements conducted in the network with an average site spacing of $1.2 \mathrm{~km}$ report the $67 \%$ CERP at a level of $170 \mathrm{~m}-190 \mathrm{~m}$. The presented accuracy figures were achieved by assessing the PCM positioning in the same weather conditions as the database was generated. Evaluation of the weather impact on the PCM accuracy is indicated for future work. However, it is expected that the eventual decrease in the accuracy will not be significant.

Other performance indicators such as the latency do not introduce any notable limits in the entire functionality of the proposed methods. The overall latency of the ECID+RTT is defined by the duration of the FSHO algorithm. In the case when the located UE needs to be forced to SHO, the latency does not surpass $2 \mathrm{~s}$ otherwise the overall response time is naturally faster. Moreover, as duration of the RTT measurement should not exceed $100 \mathrm{~ms}$, repetition of RTT measurements for reliability improvement in a multipath environment does not cause any evident delay [45]. Hence, the higher level of interference during the FSHO procedure does not affect the overall network capacity significantly. Performed measurements in an indoor UMTS network indicate that if location requests from the terminals under the same cell arrive every 30 seconds, the mean capacity during an hour is decreased by approximately $8.5 \%$. However, the instantaneous capacity drops might be significant. Therefore, it is advisable to schedule FSHO executions if multiple location requests arrive simultaneously, in order to avoid notable capacity reductions. Similarly, the duration of the PCM positioning is not large, due to complexity maintained at the lowest possible level.

Summarizing, the proposed methods are feasible for direct deployment in existing UMTS networks, without requiring any major and time consuming changes to the network equipment that involve investments exceeding a rational range. In the long term, when the AGPS technology will be supported by the majority of networks and terminals, the ECID+RTT or PCM can be utilized in hybrid satellitecellular positioning systems.

\section{ACKNOWLEDGMENTS}

Authors would like to thank Elisa Networks Oyj for enabling the measurement campaigns, Nemo Technologies Ltd. for providing a measurement tool, European Communication Engineering (ECE) Ltd. for helpful comments, and National Technology Agency of Finland for funding the work.

\section{REFERENCES}

[1] 3GPP TS 25.305, "UMTS; UE positioning in Universal Terrestrial Radio Access Network (UTRAN); Stage 2," ver. 7.1.0, Rel. 7, http://www.3gpp.org.

[2] J. Borkowski, J. Niemelä, and J. Lempiäinen, "Enhanced performance of Cell ID+RTT by implementing forced soft handover algorithm," in Proceedings of the 60th IEEE Vehicular Technology Conference, vol. 5, pp. 3545-3549, Los Angeles, Calif, USA, September 2004.

[3] J. Borkowski and J. Lempiäinen, "Geometrical transformations as an efficient mean for reducing impact of multipath propagation on positioning accuracy," in Proceedings of the 5th IEE International Conference on $3 G$ Mobile Communication Technologies, pp. 368-372, London, UK, October 2004.

[4] J. Borkowski and J. Lempiäinen, "Pilot correlation method for urban UMTS networks," in Proceedings of the 11th European Wireless Conference, vol. 2, pp. 465-469, Nicosia, Cyprus, April 2005.

[5] FCC publications, "FCC Acts to Promote Competition and Public Safety in Enhanced Wireless 911 Services," http://www. fcc.gov/Bureaus/Wireless/News_Releases/1999/nrwl9040.doc.

[6] H. Koshima and J. Hoshen, "Personal locator services emerge," IEEE Spectrum, vol. 37, no. 2, pp. 41-48, 2000.

[7] 3GPP TS 22.071, “Location Services (LCS); Stage 2,” ver. 7.3.0, Rel. 7, http://www.3gpp.org.

[8] S. Naghian, "Hybrid predictive handover in mobile networks," in Proceedings of the 58th IEEE Vehicular Technology Conference, vol. 3, pp. 1918-1922, Orlando, Fla, USA, October 2003.

[9] H.-P. Lin, R.-T. Juang, and D.-B. Lin, "Improved locationbased handover algorithm for mobile cellular systems with verification of GSM measurements data," in Proceedings of the 60th IEEE Vehicular Technology Conference, vol. 7, pp. 51705174, Los Angeles, Calif, USA, September 2004. 
[10] A. Markopoulos, P. Pissaris, S. Kyriazakaos, and E. Sykas, “Optimized handover procedure based on mobile location in cellular systems," in Proceedings of the 14th International Symposium on Personal Indoor and Mobile Radio Communications (PIMRC '03), vol. 3, pp. 2490-2494, Beijing, China, September 2003.

[11] 3GPP TS 25.215, "UMTS; Physical layer; Measurements (FDD)," ver. 6.0.0, Rel. 6, http://www.3gpp.org.

[12] L. Zhu and J. Zhu, "Signal-strength-based cellular location using dynamic window-width and double-averaging algorithm," in Proceedings of the 52nd IEEE Vehicular Technology Conference, vol. 6, pp. 2992-2997, Boston, Mass, USA, September 2000.

[13] M. A. Spirito and A. G. Mattiolli, "Preliminary experimental results of a GSM mobile phones positioning system based on timing advance," in Proceedings of the 50th IEEE Vehicular Technology Conference, vol. 4, pp. 2072-2076, Amsterdam, The Netherlands, September 1999.

[14] M. I. Silventoinen and T. Rantalainen, "Mobile station emergency locating in GSM," in Proceedings of the IEEE International Conference on Personal Wireless Communications (ICPWC '96), pp. 232-238, New Delhi, India, February 1996.

[15] J. Borkowski, J. Niemelä, and J. Lempiäinen, "Performance of Cell ID+RTT hybrid positioning method for UMTS radio networks," in Proceedings of the 5th European Wireless Conference, pp. 487-492, Barcelona, Spain, February 2004.

[16] 3GPP TSG-RAN WG1 doc. No R1-99b79, "Time Aligned IP-DL positioning technique," 1999, http://www.3gpp.org/ftp/ tsg_ran/WG1_RL1/TSGR1_07/Docs/Pdfs/R1-99b79.pdf.

[17] B. Ludden and L. Lopes, "Cellular based location technologies for UMTS: a comparison between IPDL and TA-IPDL," in Proceedings of the 51st IEEE Vehicular Technology Conference, vol. 2, pp. 1348-1353, Tokyo, Japan, May 2002.

[18] 3GPP TSG-RAN WG1 doc. No R1-00-1186, "Initial Simulation Results of the OTDOA-PE positioning method," 2000, http://www.3gpp.org/ftp/tsg_ran/WG1_RL1/TSGR1_16/Docs/ PDFs/R1-00-1186.pdf.

[19] D. Bartlett and P. Morris, "CVB: a technique to improve OTDOA positioning in $3 \mathrm{G}$ networks," Cambridge Positioning System Ltd company whitepaper, 2002, http://www.cursorsystem.com/document_library/CPS_CVB_Overview_02May 02.pdf.

[20] Project report Emily IST 2000-26040 deliverable D18, "Business Models Report,” 2002, http://www.emilypgm.com/acrobat/emily_d18.pdf.

[21] P. J. Duffett-Smith and P. Hansen, "Precise time transfer in a mobile radio terminal," Cambridge Positioning Systems Ltd company whitepaper, 2002, http://www.cursor-system.com/ cps/pdf/EGPSwhitepaper.pdf.

[22] S. Sakagami, S. Aoyama, K. Kuboi, S. Shirota, and A. Akeyama, "Vehicle position estimates by multibeam antennas in multipath environments," IEEE Transactions on Vehicular Technology, vol. 41, no. 1, pp. 63-68, 1992.

[23] J. Caffery Jr. and G. L. Stuber, "Subscriber location in CDMA cellular networks," IEEE Transactions on Vehicular Technology, vol. 47, no. 2, pp. 406-416, 1998.

[24] P. Deng and P. Z. Fan, "An AOA assisted positioning system," in Proceedings of the International Conference on Communications Technology (ICCT '00), vol. 2, pp. 1501-1504, Beijing, China, August 2000.

[25] S. Venkatraman and J. Caffery Jr., "Hybrid TOA/AOA techniques for mobile location in non-line-of-sight environments," in Proceedings of the IEEE Wireless Communications and Networking Conference (WCNC '04), vol. 1, pp. 274-278, Atlanta, Ga, USA, March 2004.
[26] L. Cong and W. Zhuang, "Hybrid TDOA/AOA mobile user location for wideband CDMA cellular systems," IEEE Transactions on Wireless Communications, vol. 1, no. 3, pp. 439-447, 2002.

[27] N. J. Thomas, D. G. M. Cruickshank, and D. I. Laurenson, "Performance of a TDOA-AOA hybrid mobile location system," in Proceedings of the 2nd IEE International Conference on $3 G$ Mobile Communication Technology, pp. 216-220, London, UK, March 2001.

[28] H. Laitinen, J. Lahteenmaki, and T. Nordstrom, "Database correlation method for GSM location," in Proceedings of the 53rd IEEE Vehicular Technology Conference, vol. 4, pp. 2504-2508, Rhodes, Greece, May 2001.

[29] O. Sallent, R. Agusti, and X. Calvo, "A mobile location service demonstrator based on power measurements," in Proceedings of the 60th IEEE Vehicular Technology Conference, vol. 6, pp. 4096-4099, Los Angeles, Calif, USA, September 2004.

[30] D. Zimmermann, J. Baumann, M. Layh, F. Landstorfer, R. Hoppe, and G. Wölfle, "Database correlation for positioning of mobile terminals in cellular networks using wave propagation models," in Proceedings of the 60th IEEE Vehicular Technology Conference, vol. 7, pp. 4682-4686, Los Angeles, Calif, USA, September 2004.

[31] S. Ahonen and H. Laitinen, "Database correlation method for UMTS location," in Proceedings of the 57th IEEE Vehicular Technology Conference, vol. 4, pp. 2696-2700, Jeju, South Korea, April 2003.

[32] S. Soliman, P. Agashe, I. Fernandez, A. Vayanos, P. Gaal, and M. Oljaca, "gpsOne ${ }^{\mathrm{TM}}$ : a hybrid position location system," in Proceedings of the 6th IEEE International Symposium on Spread Spectrum Techniques and Applications, vol. 1, pp. 330-335, Parsippany, NJ, USA, September 2000.

[33] F. van Diggelen, "Global Locate Indoor GPS Chipset \& Services," Global Locate Inc. company whitepaper, http://www. globallocate.com/GlobalLocateIndoorGPS.pdf.

[34] 3GPP TR 23.835 Release 6, "Technical Specification Group Applicability of GALILEO in LCS; Study into Applicability of GALILEO in LCS," ver. 1.0.0, Rel. 6, http://www.3gpp.org.

[35] 3GPP TR 25.922, "UMTS; Radio resource management strategies," ver. 6.0.1, Rel. 6, http://www.3gpp.org.

[36] J. Niemelä and J. Lempiäinen, "Impact of the base station antenna beamwidth on capacity in WCDMA cellular networks," in Proceedings of the 57th IEEE Vehicular Technology Conference, vol. 1, pp. 80-84, Jeju, South Korea, April 2003.

[37] G. Beveridge and R. Schechter, Optimisation: Theory and Practice, McGraw-Hill, New York, NY, USA, 1970.

[38] E. D. Kaplan, Understanding GPS: Principles and Applications, Artech House, London, UK, 1996.

[39] Y. Jeong, H. You, and C. Lee, "Calibration of NLOS error for positioning systems," in Proceedings of the 53rd IEEE Vehicular Technology Conference, vol. 4, pp. 2605-2608, Rhodes, Greece, May 2001.

[40] L. J. Greenstein, V. Erceg, Y. S. Yeh, and M. V. Clark, "A new path-gain/delay-spread propagation model for digital cellular channels," IEEE Transactions on Vehicular Technology, vol. 46, no. 46, pp. 477-485, 1997.

[41] E. S. Sousa, V. M. Jovanovic, and C. Daigneault, "Delay spread measurements for the digital cellular channel in Toronto," IEEE Transactions on Vehicular Technology, vol. 43, no. 4, pp. 837-847, 1994.

[42] J. van Rees, "Measurements of the wide-band radio channel characteristics for rural, residential, and suburban areas," IEEE Transactions on Vehicular Technology, vol. 36, no. 1, pp. 2-6, 1987. 
[43] J. Niemelä, J. Borkowski, and J. Lempiäinen, "Using IDLE mode measurements for network plan verification in WCDMA," in Proceedings of the 8th IEEE International Symposium on Wireless Personal Multimedia Communications (WPMC'05), Aalborg, Denmark, September 2005.

[44] 3GPP TR 25.853, "UMTS; Technical Specification Group Radio Access Network; Delay Budget within the Access Stratum," ver. 4.0.0, Rel. 4, http://www.3gpp.org.

[45] 3GPP TS 25.133, "UMTS; Requirements for support of radio resource management (FDD)," ver. 6.9.0, Rel. 6, http://www.3gpp.org.

[46] H. Holma and A. Toskala, WCDMA for UMTS, John Wiley \& Sons, New York, NY, USA, 3rd edition, 2004.

[47] J. Lacki, "Optimization of soft handover parameters for UMTS network in indoor environment," M.S. thesis, Tampere University of Technology, Tampere, Finland, December 2005.

Jakub Borkowski was born in Szczecin, Poland in 1980. He received the M.S. degree in information technology from Tampere University of Technology (TUT), Tampere, Finland in 2004. Currently, he is a Postgraduate student working towards the Dr. Tech. degree at Tampere University of Technology. He also works as a mobile network Senior Specialist at Teleware Oy, Finland. Before Teleware, he worked three years

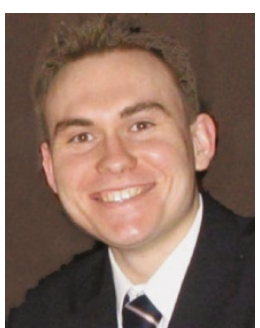
for Tampere University of Technology as a Scientist Researcher. His main interests include UMTS radio network planning and development of cellular location techniques.

Jukka Lempiäinen was born in Helsinki, Finland, in 1968. He received the M.S., Lic. Tech., and Dr. Tech. degrees, all in electrical engineering, from Helsinki University of Technology, Espoo, Finland, in 1993, 1998, and 1999 , respectively. He is a Senior Partner and President of European Communications Engineering (ECE) Ltd. Before ECE Ltd., he worked more than five years in Nokia in different positions in the area of

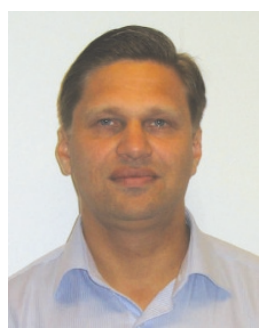
radio network planning and he has altogether more than 12 years experience in GSM-based mobile network planning and consulting. Currently, he is also a Professor in Tampere University of Technology, Finland. He has concentrated on the radio planning of the cellular networks during his whole career. His main interests are to combine the coverage and capacity related topics (topology planning in UMTS) and to adjust the performance of the technical details like diversity reception and GPRS traffic for the air interface. He has three patents. He is a URSI National Board Member, Finland. 